\title{
ULTIMATE-Subaru: Wide-field Near-infrared Surveyor with GLAO at Subaru telescope
}

Yosuke Minowa*a ${ }^{* a}$ Christophe Clergeon ${ }^{\mathrm{a}}$, Masayuki Akiyama ${ }^{\mathrm{b}}$, Francois Rigaut ${ }^{\mathrm{c}}$, Celine d'Orgeville ${ }^{\mathrm{c}}$, Ian Price ${ }^{\mathrm{c}}$, Nick Herrald ${ }^{\mathrm{c}}$, Yusei Koyama ${ }^{\mathrm{a}}$, Ikuru Iwata ${ }^{\mathrm{a}}$, Takashi Hattori ${ }^{\mathrm{a}}$, Tadayuki Kodama ${ }^{\mathrm{b}}$, Yutaka Hayano ${ }^{\mathrm{d}}$, Shin Oya ${ }^{\mathrm{d}}$, Ichi Tanaka ${ }^{\mathrm{a}}$, Kentaro Motohara ${ }^{\mathrm{e}}$, Nobuo Arimoto ${ }^{\mathrm{f}}$, and Michitoshi Yoshida ${ }^{\mathrm{a}}$

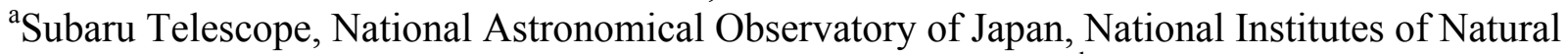
Sciences (NINS), 650 North A'ohoku Place, Hilo, HI, 96720, USA; ${ }^{b}$ Astronomical Institute, Tohoku

University, Aramaki, Aoba-ku, Sendai 980-8578, Japan; ${ }^{\mathrm{c}}$ The Australian National University, RSAA, Mount Stromlo Observatory, Cotter Road, Weston Creek, ACT 2611, Australia; ${ }^{\mathrm{d}}$ National

Astronomical Observatory of Japan, 2-21-1 Osawa, Mitaka, Tokyo, 181-8588, Japan;

${ }^{\mathrm{e}}$ Institute of Astronomy, University of Tokyo, 2-21-1 Osawa, Mitaka, Tokyo 181-0015, Japan;

${ }^{\mathrm{f}}$ Astronomy Program, Department of Physics and Astronomy, Seoul National University 599

Gwanak-ro, Gwanak-gu, Seoul, 151-742, Korea

\begin{abstract}
ULTIMATE-Subaru is a next large facility instrument program at Subaru telescope, which will develop $14 \times 14 \operatorname{arcmin}^{2}$ wide-field near-infrared imager and multi-object spectrograph with the aid of ground-layer adaptive optics system (GLAO) at the Cassegrain and Nasmyth foci of the telescope in mid 2020s. Our preliminary performance simulation indicates that the GLAO system at Subaru telescope can provide uniform seeing improvement down to FWHM 0.2 arcsec in K band under moderate seeing condition across the field of view up to $20 \mathrm{arcmin}$ in diameter. The main science case of the ULTIMATE-Subaru is a complete census of galaxy evolution from cosmic dawn ( $\mathrm{z}>8)$ to cosmic noon $(\mathrm{z}=1-3)$ with ultradeep, wide-field narrow-band imaging and multi-object spectroscopies of a statistical sample of galaxies especially in $K$ band.
\end{abstract}

Keywords: instrumentation: adaptive optics

\section{INTRODUCTION}

In the next decade, the Subaru telescope will offer unique wide-field imaging and spectroscopic capabilities at the prime focus using Hyper Suprime Cam ${ }^{1}$ (HSC) and Prime Focus Spectrograph ${ }^{2}$ (PFS). These instruments provide superb survey capabilities mainly in optical wavelength range and occupy the most of dark nights at the Subaru telescope. To further strengthen the wide-field capability of the Subaru Telescope, we have initiated ULTIMATE-Subaru project since 2011. The ULTIMATE-Subaru stands for Ultra-wide Laser Tomographic Imager and Multi-object spectrograph with AO for Transcendent Exploration by Subaru. We aim at developing wide-field near-infrared (NIR) imager and multi-object spectrograph (or IFU spectrograph) assisted by a ground-layer adaptive optics (GLAO) system as a new facility instrument of Subaru. The GLAO system will uniformly improve image quality over wide field of view ( 20 arcmin) by correcting for the turbulence at the ground layer of the Earth's atmosphere. While our unique wide-field instruments (HSC and PFS) are optical instruments and they are operated in dark nights, ULTIMATE-Subaru will make full use of bright nights of the Subaru Telescope in 2020s. Based on our simulation (as described in Section 3 in more details), the ULTIMATE-Subaru GLAO system can provide FWHM 0.2 arcsec imaging quality over $\sim 20$ arcmin field of view at K-band under moderate seeing conditions at Maunakea. It is worth noting that the spatial resolution achieved by ULTIMATE-Subaru is comparable to that of the Hubble Space Telescope in NIR, as well as the future WFIRST which will be launched in mid 2020s. The improvement in the image quality will also enable to improve the sensitivity by a factor of $\sim 1.5-2.0$, which will greatly improve the survey power, particularly for faint compact sources in the distant universe. We published a study report ${ }^{3}$ of the ULTIAMTE-Subaru project in January 2016. This document summarizes science cases, AO/instrument design studies, as well as our development plans. In this paper, we introduce the conceptual design of the ULTIMATE-Subaru project based on the study report. The layout of this paper is as follows. In Section 2, we describe an overview of the ULTIMATE-

*minoways@naoj.org; phone +1-808-934-5905 
Subaru GLAO system and wide-field NIR instruments. We also introduce the expected performance of the ULTIMATESubaru GLAO system in Section 3. Science cases for the ULTIMATE-Subaru are given in Section 4. Finally, we will briefly show the current activities on the ULTIMATE-Subaru conceptual design and schedule of the project in Section 5.

\section{INSTRUMENT OVERVIEW}

The ULTIMATE-Subaru project will develop wide-field near-infrared imager and multi-object spectrograph assisted by ground-layer adaptive optics (GLAO) system as a Subaru's next facility instrument. Figure 1 shows the schematic overview of the ULTIMATE-Subaru subsystems. The GLAO system consists of an adaptive secondary mirror (ASM), four laser guide stars (LGSs) launched from the side of the telescope, four LGS wavefront sensors, and three or more natural guide star wavefront sensors for low-order tip/tilt and focus correction. The existing secondary mirror will be replaced by the ASM with 1000 actuators. Baseline specification of the ULTIMATE-Subaru GLAO system is summarized in Table 1. One of the unique capabilities of the ULTIMATE-Subaru is a large field coverage area of up to 20 arcmin in diameter. At the Cassegrain focus, we found that the maximum 20 arcmin diameter field-of-view (FoV) can be cleared by removing the peripheral optical components, such as atmospheric dispersion corrector, slit viewer, calibration light source, and auto-guider and Shack-Hartmann camera for primary mirror analysis. Since such components are not necessary for the ULTIMATE-Subaru, we will remove them to get the maximum FoV. The patrol field of regard of the wavefront sensors will be at outside of the science FoV, which is $14 \mathrm{x} 14$ square arcmin.

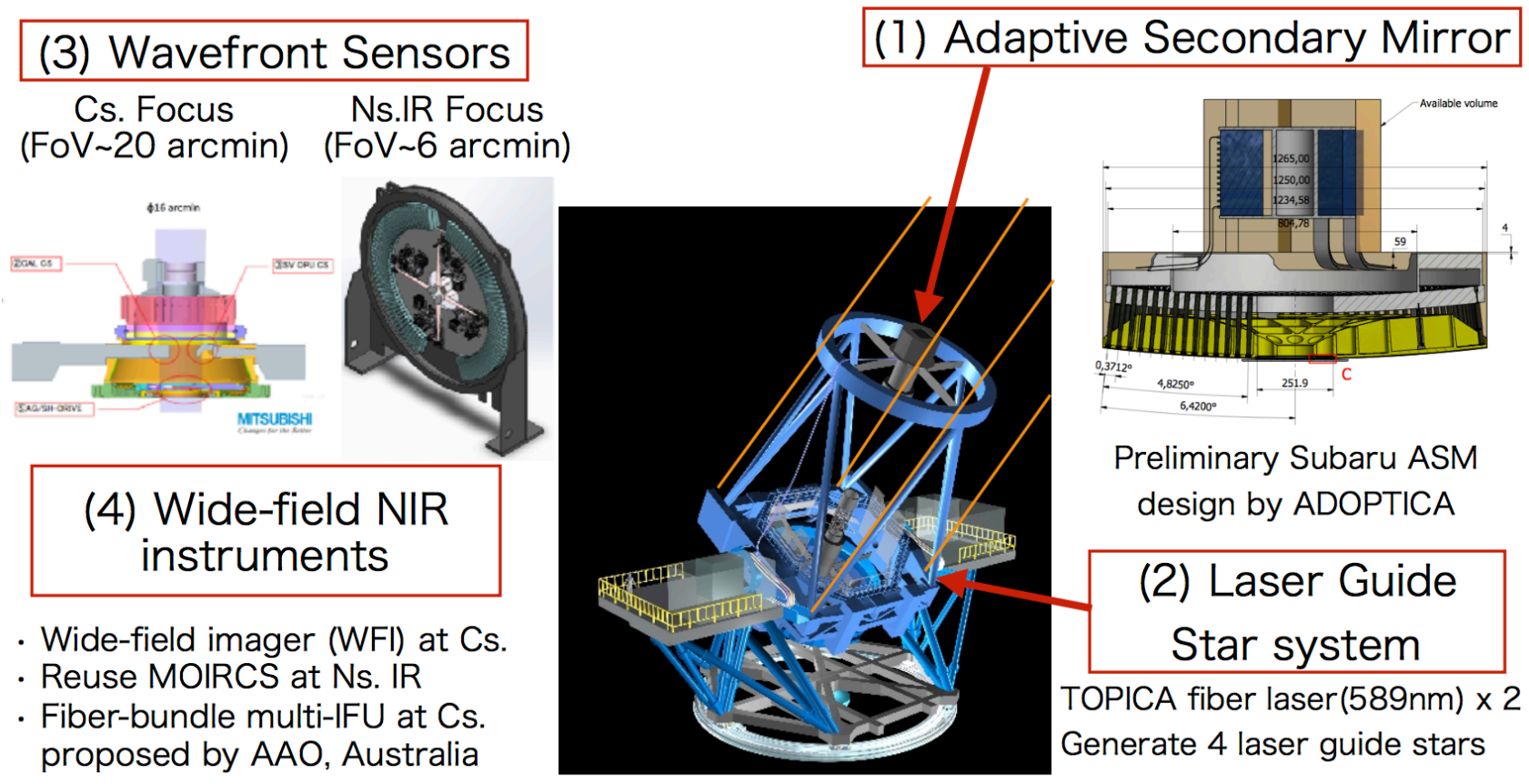

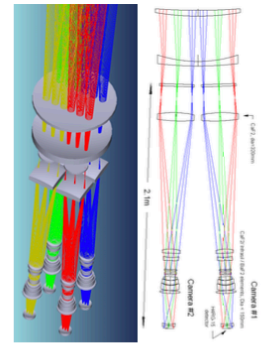

Imager concept

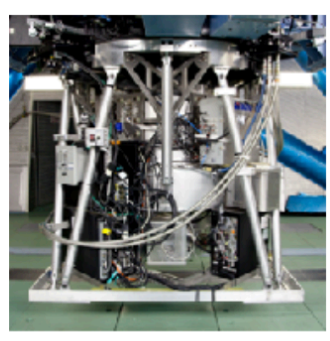

MOIRCS

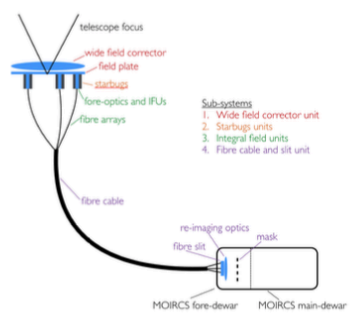

Multi-IFU concept

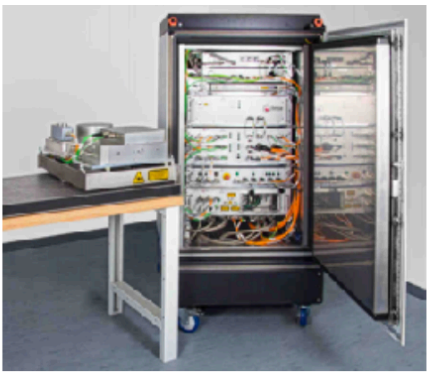

Figure 1. A schematic view of the ULTIMATE-Subaru subsystems for wide-field near-infrared $(0.9-2.4 \mu \mathrm{m})$ imager and spectrograph with the aid of ground-layer adaptive optics. 
Table 1. Baseline specifications of the ULTIMATE-Subaru AO system at the Cassegrain focus.

\begin{tabular}{|l|l|}
\hline \multicolumn{1}{|c|}{ Item } & \multicolumn{1}{c|}{ Specification } \\
\hline Guide stars & 4 LGS, 2 4 NGS \\
\hline Location of guide stars & $\begin{array}{l}\left.\text { LGS: Edge of FoV (r } \sim 10^{\prime}\right), \\
\text { NGS: } r<10^{\prime}, \text { Outside of science FoV (14'x14') }\end{array}$ \\
\hline LGS wavefront sensor & Shack-Hartmann \\
\hline Tip/tilt wavefront sensor & $2 \times 2$ Shack-Hartmann or Pyramid \\
\hline Number of sub-apertures & $>100$ \\
\hline Frame rate of wavefront sensor & $>500 H z$ \\
\hline Deformable mirror & Adaptive secondary mirror with $~ 1000$ actuators \\
\hline Actuators & $\sim 1000$ \\
\hline AO control type & GLAO (LTAO and ExAO are optional) \\
\hline
\end{tabular}

We have been conducting the conceptual studies of the ULTIMATE-Subaru NIR instruments: wide-field imager, multi-object slit (MOS) spectrograph, and multi-object integral field unit (IFU) spectrograph, which fully utilize the capabilities of the GLAO corrected spatial resolution and the wide FoV up to 14 x 14 square arcmin. We are considering the possibility to reuse the existing wide-field multi-object imager and spectrograph MOIRCS ${ }^{4}$ at the Nasmyth platform as a first light instrument for the ULTIMATE-Subaru. We are also considering the development of a wide-field NIR imager (WFI) at the Cassegrain focus, which has 14 x 14 square arcmin science FoV, as a workhorse instrument for a large near-infrared imaging survey program with the ULTIMATE-Subaru. To fulfill the requirement from the science cases with the ULTIMATE-Subaru, we are planning to install a wide-variety of narrow/medium band filters especially in $K$ band as well as regular broad-band filter set $(Y J H K)$ in NIR. The conceptual design of the multi-object IFU spectrograph (M-IFU) has been conducted in collaboration with Australian Astronomical Observatory (AAO) $)^{5}$. The conceptual design assumes 8-13 fiber bundle IFUs with 1".18 x 1". $18 \mathrm{FoV}$ for each, which are attached underneath of the wide-field corrector optics installed at the Cassegrain focus and are deployable anywhere on the focal plane at the Cassegrain focus using robotic positioners, called "Starbug", developed by AAO. The fibers from the IFUs will be connected to any near-infrared spectrograph, although we consider MOIRCS spectrograph mode in the conceptual study. If we feed the light from the Starbug positioners into PFS spectrographs, which consist of 4 fiber-fed spectrograph modules with 2,394 optical fibers, we can increase the number of the IFUs up to $\sim 40$, providing a unique kinematic survey capability at distant universe ( $\mathrm{z}>1$ ) like $\mathrm{MANGA}^{6} / \mathrm{SAMI}^{7}$ surveys at $\mathrm{z} \sim 0$.

\section{PERFORMANCE EXPECTATION}

GLAO performance simulations have been conducted by Oya et al. $(2012,2014)^{8,9}$ using the MAOS simulation package $^{10}$. Figure 2 shows the probability distributions of seeing-limited and GLAO corrected PSF size in $H$ and $K$ band. The expected image quality after the GLAO correction at Subaru is FWHM $\sim 0.2 \operatorname{arcsec}$ in $K$-band under a moderate seeing condition of FWHM $\sim 0.4$ arcsec over $\sim 20$ arcmin FoV. We confirmed that the results of the simulation in $H$ band is consistent with the on-sky results by RAVEN ${ }^{11}$ at the Subaru telescope in GLAO mode ${ }^{12}$, demonstrating the feasibility to achieve the performance of the GLAO as expected. To predict the sensitivity of the ULTIMATE-Subaru NIR instruments, we conducted the performance modeling of the instruments using the PSF obtained from the GLAO simulation. Our performance simulation results suggest that the seeing improvement by GLAO in $K$ band will provide 2.5-3.0 times higher sensitivity for point sources and $\sim 1.5$ times higher sensitivity for extended sources (assuming highredshift galaxies with the effective radius of $0 " .25$, or $2 \mathrm{kpc}$ at $\mathrm{z} \sim 2$ ) than the seeing-limited observations. The combination of the sensitivity improvement by GLAO and the wide-field coverage by the ULTIMATE-Subaru NIR instruments will provide unique and strong survey capability at the Subaru telescope. Efficiency of the wide-field 
imaging survey with the ULTIMATE WFI is expected to be 20 times higher than the current MOIRCS under seeing limited condition.
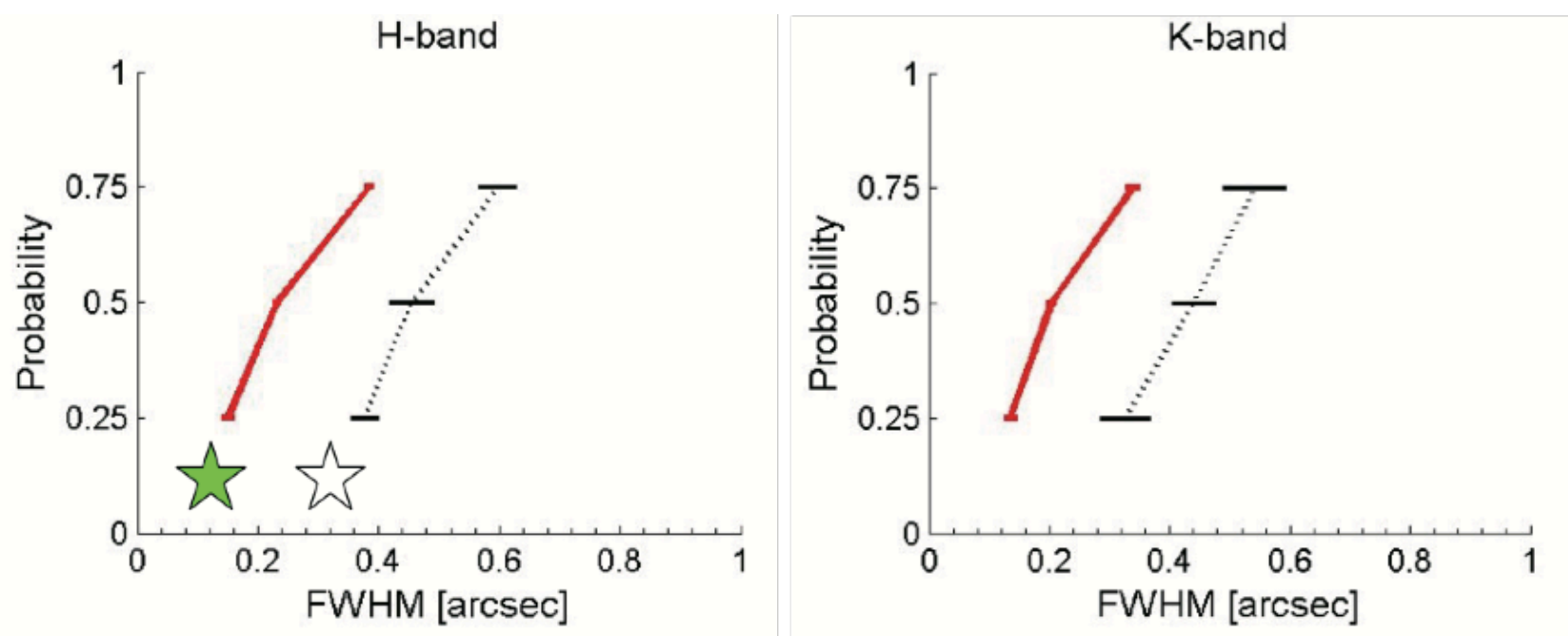

Figure 2. Probability distributions of the PSF size (FWHM in arcsec) in $H$ (left) and $K$ (right) band for seeing-limited (black dotted lines) and GLAO assisted (red solid lines) observations. The star symbols in the left panel show the on-sky results by RAVEN used in GLAO mode. Filled and open symbols show the PSF size with and without GLAO correction, respectively.

\section{SCIENCE CASE}

The wide-field survey power and multiplicity together with the high spatial resolution achieved by ULTIMATE-Subaru allows us to conduct unprecedentedly deep and wide-field NIR extra-galactic survey (Figure 3). This is an essential step for us to prepare for the era of $30 \mathrm{~m}$ class telescopes by having our original, excellent targets. In particular, ultra-deep, wide-field narrow-band and medium-band imaging will be the most unique part of the ULTIMATE-Subaru. Narrowband imaging will allow us to find very distant galaxy candidates at $\mathrm{z}>8$ based on Ly $\alpha$ emission. This is a direct extension of the current Subaru science (with Suprime-Cam and HSC) towards more distant universe. Narrow-band imaging with high spatial resolution will also tell us where in galaxies they are forming new stars, how the star-forming activity propagates with cosmic times, mass, and environment. We note that the future wide-field NIR space missions (Euclid and WFIRST) do not cover $\lambda>2 \mu \mathrm{m}$, and therefore ULTIMATE-Subaru can largely complement those space missions by conducting large survey in $K$ band. By splitting the $K$ band into three medium bands, we can select Balmer break galaxies to the $\mathrm{z} \sim 5$, which will revolutionize our understanding of the distant universe currently explored with rest-frame UV light alone. Deep spectroscopic survey of high-z galaxies $(1<\mathrm{z}<3.5)$ at K-band is also unique, and kinematics along the slits based on the line profiles and velocity offsets will tell us how the turbulent disks and galactic central bulges are formed, and star formation is quenched. Furthermore, multi-object IFU survey assisted by GLAO for a large sample of galaxies at the cosmic noon $(1<\mathrm{z}<3.5)$ will tell us the internal physical properties such as spatial distributions of dust extinction, ionizing states and gaseous metallicities for a statistical sample of galaxies for the first time. In addition to the high-redshift galaxy science, the expected performance of ULTIMATE-Subaru will also be useful for a wide variety of science cases for nearby galaxies $(z<0.3)$, the Galactic center, globular clusters, and Galactic star-forming regions.

\section{SCHEDULE AND CURRENT ACTIVITIES}

Currently, we are conducting conceptual design and feasibility studies of the ULTIMATE-Subaru project. The conceptual design of the GLAO system has been conducting in collaboration with Tohoku University and Australian National University. The specification of the GLAO system is being optimized through the extensive performance simulation by varying the ground layer turbulence based on the statistical measurements of the Mauna Kea ground layer turbulence profile ${ }^{13}$. Once the specifications of the GLAO are fixed, we will conduct the conceptual optical and mechanical design of the wavefront sensor systems and LGS facility. Next milestone for the ULTIMATE-Subaru project 
is to have a conceptual design review of the GLAO system in mid-2018. We are aiming to have first light of the GLAO in mid-2020s.

In addition to the conceptual design, we are also conducting the upgrade of the existing facility AO system (AO188) at the Subaru telescope to develop fundamental technologies for the ULTIMATE-Subaru GLAO system. Currently, we are developing a new real-time control system for AO188 that will be able to use for the future GLAO system. The TOPTICA fiber laser $(589 \mathrm{~nm})$ system, which will be used for the GLAO system as well, is being delivered to the Subaru telescope for upgrading the current LGS facility used by AO188 in LGS mode. We are going to develop laser beam diagnostics and steering system so that we can use the same system for the future ULTIMATE-Subaru GLAO system. We are also planning to implement a laser tomographic AO (LTAO) mode in AO188 to improve the performance of AO188 especially in optical wavelength and to demonstrate tomographic wavefront reconstruction with multiple laser guide stars, which is also essential for the ULTIMATE-Subaru GLAO system. The 4 Shack-Hartmann wavefront sensor system to conduct laser tomography with 4 laser guide star system is being designed. The same design will be used for the ULTIMATE GLAO system. We are currently prototyping the Shack-Hartmann wavefront sensor system to validate the design.

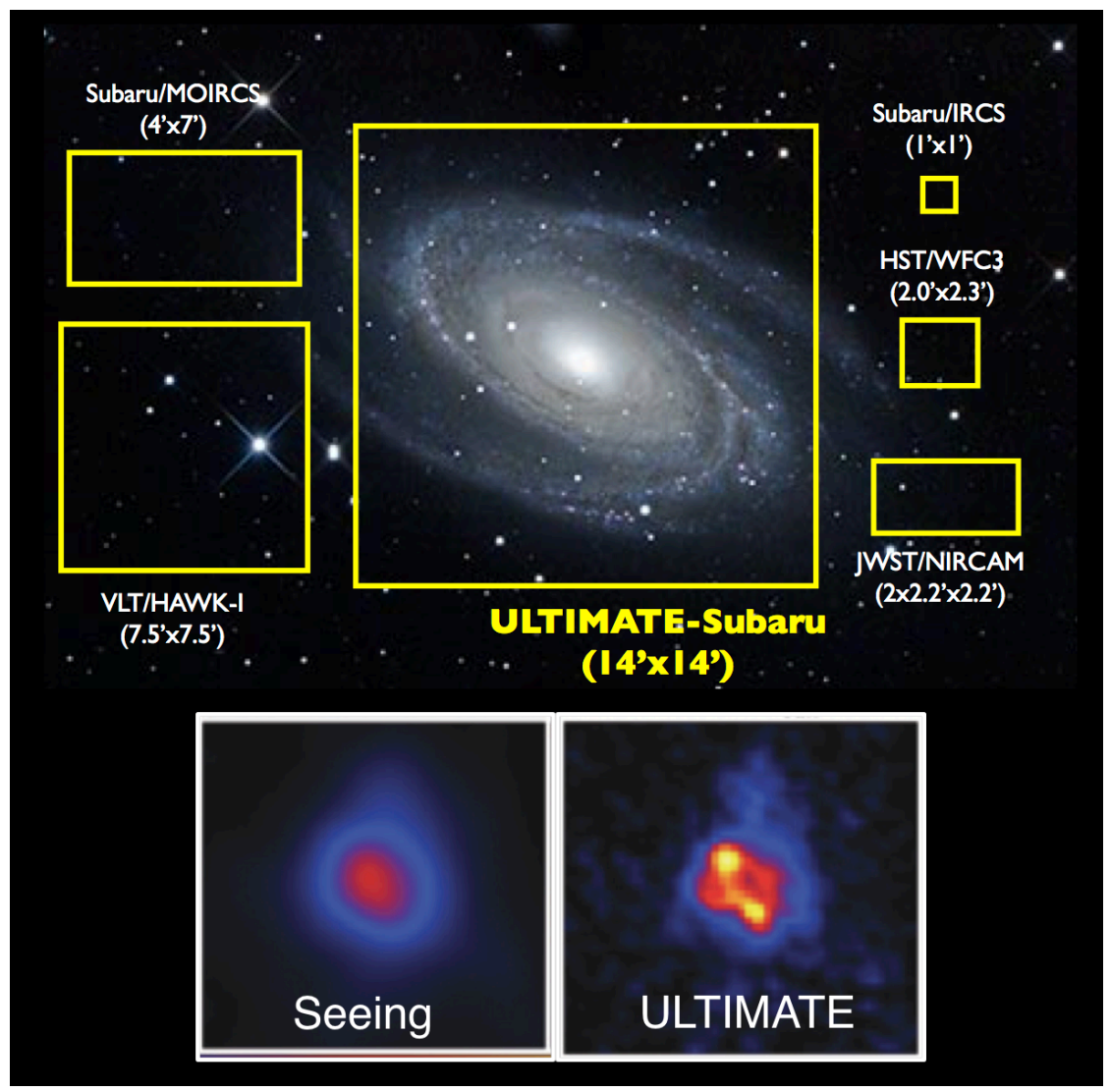

Figure 3. (Top): Comparison of the current and future NIR facilities accessible to $\lambda>2 \mu \mathrm{m}$. (Bottom): A simulated $\mathrm{z} \sim 2$ galaxy, demonstrating the importance of GLAO correction to reveal their internal structures.

\section{REFERENCES}

[1] Miyazaki, S., Komiyama, Y., Nakaya, H., Kamata, Y., and Doi, Y. et al., "Hyper Suprime-Cam," in [Ground-based and Airborne Instrumentation for Astronomy IV], Proc. SPIE 8446, 84460Z (2012).

[2] Tamura, N., Takato, N., Shimono, A., Moritani, Y., and Yabe, K. et al., "Prime Focus Spectrograph (PFS) for the Subaru Telescope: Overview, recent progress, and future perspectives," in [Ground-based and Airborne Instrumentation for Astronomy VI], Proc. SPIE 9908, 99081M (2016). 
[3] ULTIMATE-Subaru working group \& science team, "ULTIMATE-Subaru Study Report 2016." 13 January 2016, http://www.naoj.org/Projects/newdev/ngao/20160113/index.html.

[4] Suzuki, R., Tokoku, C., Ichikawa, T., Uchimoto, Y. K., and Konishi, M. et al., "Multi-Object Infrared Camera and Spectrograph (MOIRCS) for the Subaru Telescope I. Imaging", PASJ, 60, 1347 (2008).

[5] Ellis, S., Zhelem, R., Brown, D. M., Staszak, N. F., and Lidman, C. et al., "ULTIMATE: a deployable multiple integral field unit for Subaru," in [Ground-based and Airborne Instrumentation for Astronomy VI], Proc. SPIE 9908, 99081Q (2016).

[6] Bundy, K., Bershady, M. A., Law, D. R., Yan, R., Drory, N. et al., "Overview of the SDSS-IV MaNGA Survey: Mapping nearby Galaxies at Apache Point Observatory”, ApJ, 798, 7 (2015).

[7] Bryant, J. J., Owers, M. S., Robotham, A. S. G., Croom, S. M., Driver, S. P. et al., "The SAMI Galaxy Survey: instrument specification and target selection", MNRAS, 447, 2857 (2015).

[8] Oya, S., Akiyama, M., Hayano, Y., Minowa, Y., and Iwata, I. et al., "A preliminary simulation result of the nextgeneration wide-field AO at Subaru Telescope", in [Adaptive Optics Systems III], Proc. SPIE 8447, 84473V (2012).

[9] Oya, S., Hayano, Y., Lai, O., Iwata, I., and Kodama, T. et al., "ULTIMATE-SUBARU: simulation update," in [Adaptive Optics Systems IV], Proc. SPIE 9148, 91486G (2014).

[10] Wang, L., http://lianqiw.github.com/maos/

[11] Lardi'ere, O., Andersen, D., Blain, C., Bradley, C., and Gamroth, D. et al., "Multi-object adaptive optics on-sky results with Raven," in [Adaptive Optics Systems IV], Proc. SPIE 9148, 91481G (2014).

[12] Ono, Y. H., Correia, C. M., Lardi 'ere, O., Andersen, D., and Oya, S. et al., "On-sky MOAO performance evaluation of RAVEN" in [Adaptive Optics Systems V], Proc. SPIE 9909, 990910 (2016).

[13] Chun, M., Wilson, R., Avila, R., Butterley, T., Aviles, J.-L. et al., "Mauna Kea ground-layer characterization campaign", MNRAS, 394, 1121 (2009). 